\title{
Mobilisation des connaissances professionnelles des enseignants dans la préparation et la mise en cuuve des cours de mathématiques à l'école fondamentale au Burundi
}

Université du Burundi (Burundi)

Karine Bécu-Robinault École normale supérieure de Lyon (France)

\author{
Mobilization of the professional knowledge of teachers in the preparation and \\ implementation of mathematics courses at the basic school in Burundi
}

doi: $10.18162 /$ fp. 2021.565

\section{ésumé}

La réforme curriculaire, initiée au Burundi en 2013 dans l'enseignement fondamental, implique d'avoir recours à des pratiques enseignantes inédites pour certains ensei-gnants non formés à l'enseignement des mathématiques. Notre étude vise à explorer comment les enseignants de mathématiques mobilisent leurs connaissances dans leurs pratiques. L'analyse des entretiens, menés auprès de 20 enseignants, montre que les enseignants suivent les prescriptions du curriculum. Les enseignants formés à l'enseignement des mathématiques s'appuient notamment sur des connaissances acquises en formation initiale, alors que les autres jouent sur leur expérience dans l'enseignement. Les perceptions des enseignants sur l'efficacité des formations re-çues jouent un rôle sur les pratiques enseignantes.

Mots-clés

Connaissances professionnelles, mathématiques, École fondamentale au Burundi.

Abstract

The curriculum reform introduced in Burundi in 2013 in basic school requires for teaching practices to which some teachers were not initially trained. Our study aims to explore how mathematics teachers mobilize their knowledge in their practices. The analysis of interviews with twenty teachers shows that teachers follow tend the cur-riculum requirements. Teachers who have been trained in mathematics education rely on knowledge acquired in initial training while others use their experience in teaching. The teachers' perceptions of the effectiveness of the training received, in-fluence teaching practices.

Keywords Professional knowledge, mathematics, basic schools in Burundi.

\section{Contexte de l'étude}

Les connaissances professionnelles nécessaires au métier d'enseignant se construisent en partie lors de la formation initiale du futur enseignant. Les savoirs professionnels acquis au cours de cette formation devraient lui permettre de s'adapter aux situations éducatives auxquelles il sera confronté (Paquay, Marguerite, Charlier et Perrenoud, 2012). Néanmoins, selon Schwille, Dembélé et Schubert (2007), cette formation initiale ne pouvant pas tout prévoir, les formations continues constituent une autre voie de développement des connaissances professionnelles (Paquay, 2007). En effet, les enseignants doivent, au fil de leur pratique professionnelle, construire de nouvelles connaissances (Wittorski et Briquet-Duhazé, 2010) pour s'adapter, par exemple, à des réformes curriculaires (Jorro, 2014). La réflexion considérée comme une métacompétence qui favorise le développement des autres compétences professionnelles en enseignement (Leroux et Vivegnis, 2019) doit caractériser les enseignants.

Pour se conformer à la communauté de l'Africaine de l'Est, dont il est membre, le Burundi a opéré en 2013 une réforme de l'enseignement de base et secondaire concernant notamment la structure et les contenus des enseignements. Dans cette étude, nous nous intéressons à l'enseignement des mathématiques au quatrième et dernier cycle de l'École fondamentale au Burundi $\left(7^{\mathrm{e}}, 8^{\mathrm{e}}\right.$ et $9^{\mathrm{e}}$ années, équivalent aux classes de $5^{\mathrm{e}}, 4^{\mathrm{e}}$ et $3^{\mathrm{e}}$ année du collège français). Les enseignants exerçant à ce niveau d'enseignement sont formés soit au secondaire, soit dans des établissements d'enseignement supérieur avec une formation à l'enseignement ou non, dans des filières mathématiques ou non. Ainsi, le profil de formation initiale des enseignants de mathématiques est extrêmement varié : mathématiques, physique, 
chimie, voire agriculture, agrobiologie, agronomie, communication, construction, électricité, informatique, psychologie, histoire, etc. (ministère de l'Éducation, de l'Enseignement supérieur et de la Recherche scientifique, 2017). Peu importe leur profil, les enseignants doivent mettre en œuvre un curriculum en rupture avec les précédents. Ce curriculum appelle des pratiques d'enseignement auxquelles bon nombre d'enseignants n'ont été ni formés ni confrontés en tant quélèves. Pour aider à la mise en œuvre de ce changement curriculaire, des formations continues obligatoires pour tous les enseignants sont organisées pendant les vacances scolaires. Cependant, dans certains pays, les recrutements massifs des enseignants risquent de compromettre la qualité de l'éducation (Djibo et Gauthier, 2017).

Notre étude se place dans un contexte de mise en œuvre d'un nouveau curriculum par des enseignants de formations initiales diverses, dont certaines ne préparent pas à l'enseignement. Notre questionnement se formule comme suit: Comment les enseignants du quatrième cycle de l'École fondamentale burundaise préparent-ils et enseignent-ils le cours de mathématiques?

Nous avons choisi de travailler à la fois sur des activités de "préparation» et de "mise en œuvre d'un cours", estimant que la classe ne demeure pas le seul lieu d'élaboration des connaissances des enseignants. Au cours du travail en amont de préparation du cours, des décisions mobilisent de manière implicite des connaissances professionnelles (Bécu-Robinault, 2007). Les pratiques d'enseignement restent idéalement contextualisées (Talbot, 2012), c'est-à-dire que celles qui sont prévues peuvent varier en classe en fonction du contexte de la classe et être réajustées (Jameau, 2015; Shing, Saat et Loke, 2015). En travaillant sur la préparation et la mise en œuvre d'un cours, nous pouvons étudier les liens établis par l'enseignant entre le curriculum réalisé et le curriculum prescrit (Demeuse, 2013; Perrenoud, 1993). Autrement dit, nous voulons savoir comment les enseignants établissent un compromis entre ce qu'on leur demande de réaliser - prescriptions officielles - et les exigences des tâches ou le contexte de leur classe - travail réel (Brau-Antony et Hubert, 2014).

\section{Cadre théorique}

Dans notre étude, nous articulons les connaissances et les pratiques enseignantes. Les pratiques correspondent dans notre cas à ce que l'enseignant réalise lors de la préparation d'un cours en dehors de la classe et à ce qu'il effectue en classe en présence de ses élèves. Les pratiques sont étudiées en relation avec les origines des connaissances mobilisées pour mettre en œuvre les orientations curriculaires. Nous considérons la notion de curriculum dans son acceptation anglo-saxonne: un plan d'action pédagogique plus large qu'un programme d'études, définissant les finalités et éclairant les orientations à donner aux activités d'enseignement, d'apprentissage et d'évaluation. Ce plan indique également le matériel didactique et les manuels scolaires à utiliser (Jonnaert, 2011).

Les recherches sur les connaissances des enseignants se sont intensifiées dans les années 1980 à la suite de la typologie de connaissances initiée par Shulman en 1986 et raffinée en 1987 dans un contexte de refonte des programmes de formation à l'enseignement. Shulman (1986) constate que les recherches insistaient jusqu'alors sur la façon dont les enseignants géraient leurs classes, organisaient les activités, structuraient le temps, planifiaient les leçons, mais oubliaient de s'interroger sur le contenu des leçons enseignées. La question qui se pose est de savoir où les enseignants trouvent les explications

2 - Formation et profession 29(2), 2021 
qu'ils donnent à leurs élèves et comment ils décident de ce qu'ils enseignent. Il est aussi question de savoir comment les enseignants interrogent et font face à des problèmes d'incompréhension chez les élèves. Il propose alors une première classification fondée sur trois bases de connaissances des enseignants: "content knowledge» (connaissances sur la discipline), "Pedagogical Content Knowledge $(P C K) »$ (connaissances pédagogiques liées au contenu disciplinaire) et enfin la catégorie qu'il a appelée «curricular knowledge» (connaissances sur le curriculum).

Dans son modèle raffiné, publié en 1987, il estime que pour que son enseignement reste efficace, tout enseignant doit avoir sept connaissances de base (Shulman, 1987) : 1) connaissances du contenu; 2) connaissances générales pédagogiques; 3) connaissance du curriculum. Il cite aussi 4) «Pedagogical Content Knowledge, $\mathrm{PCK}$; 5) connaissances des apprenants et de leurs caractéristiques; 6) connaissances des contextes éducatifs; 7) connaissances des fins, des buts et des valeurs de l'éducation et de leurs motifs philosophiques et historiques. À la suite de la catégorisation de Shulman (1986, 1987), d'autres chercheurs comme Cochran, King et DeRuiter (1991), Smith et Neale (1989) et Abell (2007) vont la compléter. Grossman (1990) a le mérite d'avoir spécifié davantage la composition des PCK, et d'autres comme Carlsen (1999), Morine-Dershimer et Kent (1999) ont précisé les catégories de connaissances déjà établies.

Dans le domaine des mathématiques, Loewenberg Ball, Thames et Phelps (2008) étudient la nature des connaissances professionnelles mathématiques pour l'enseignement. Ils se focalisent sur les PCK et sur les connaissances de contenu «pur», spécifique au travail de l'enseignement des mathématiques (p. 386). Les auteurs indiquent qu'un enseignant doit notamment avoir des connaissances mathématiques et des compétences sur l'enseignement des mathématiques. L'enseignant doit aussi anticiper ce que les élèves peuvent penser et ce qu'ils trouveront confus, prédire ce que les élèves trouveront intéressant et motivant (p. 401). L'enseignement des mathématiques demeure tributaire de leur compréhension, car cela permet à l'enseignant de savoir comment il les enseigne et d'exercer une liberté pédagogique, ce qui rejoint le point de vue de Shulman (1987) : «To teach is first to understand» (notre traduction : enseigner une discipline c'est d'abord la comprendre).

La revue de la littérature sur les modèles de connaissances des enseignants établis par différents chercheurs met en évidence l'absence d'un consensus sur ce que recouvrent ces connaissances. Quoi qu'il en soit, les connaissances consensuelles dans la communauté des chercheurs, toutes disciplines confondues, sont les connaissances sur le contenu disciplinaire, les connaissances pédagogiques, les PCK et les connaissances sur le contexte. Aucune des catégorisations précédemment citées ne peut constituer une référence unique et satisfaisante pour l'étude des connaissances des enseignants dans tous les contextes. Dans notre cas, c'est la classification des connaissances proposée par Magnusson, Krajcik, et Borko (1999) qui nous paraît la plus adaptée au contexte de mise en œuvre d'un curriculum de mathématiques à l'École fondamentale au Burundi. En effet, leur modèle met l'accent sur le rôle du contexte dans l'enseignement, et les auteurs conceptualisent davantage les PCK comme outil pour comprendre l'enseignement des sciences (les mathématiques dans notre cas). En outre, le modèle de Magnusson et al. (1999) prend en compte bien plus clairement que les autres la dimension de l'évaluation des apprentissages. Ils estiment que, pour qu'un enseignement scientifique demeure efficace, l'enseignant doit poser un certain nombre de questions : 
Que dois-je faire avec mes élèves pour les aider à comprendre ce concept scientifique? Quels matériaux sont là pour m'aider? Qu'est-ce que mes étudiants sont susceptibles de savoir déjà et quiest-ce qui sera difficile pour eux? Quelle est la meilleure façon d'évaluer ce que mes élèves ont appris? (p. 95)

Magnusson et al. (1999) distinguent ainsi quatre grands domaines de connaissances des enseignants : 1) connaissances disciplinaires et des conceptions ; 2) connaissances pédagogiques et des conceptions; 3) connaissances pédagogiques liées au contenu disciplinaires-PCK; 4) connaissances et conceptions sur le contexte (p. 98). À l'instar d'autres chercheurs, Magnusson et al. (1999) estiment que les PCK restent une catégorie de connaissances professionnelles essentielle et spécifique aux enseignants; les PCK sont liées aux capacités de l'enseignant à aider ses élèves à comprendre un sujet spécifique.

Sur la base du cadre théorique que nous venons de développer, nos questions de recherche se présentent comme suit : comment les enseignants mobilisent-ils leurs connaissances professionnelles dans la préparation et la mise en cuvre du cours de mathématiques à l'École fondamentale burundaise? D'où viennent ces connaissances?

Fondée sur la classification des connaissances de Magnusson et al. (1999), la figure 1 représente les quatre domaines de connaissances sur lesquels porte notre étude et détaille les types de connaissances de chacun de ces domaines. Dans le cadre de notre recherche, «les sciences» sont à lire comme «les mathématiques».

Connaissances disciplinaires et des conceptions (sur les mathématiques)
Connaissances pédagogiques et des conceptions (sur la gestion de la classe)

- Principes et buts de l'enseignement

- Apprenants et apprentissages

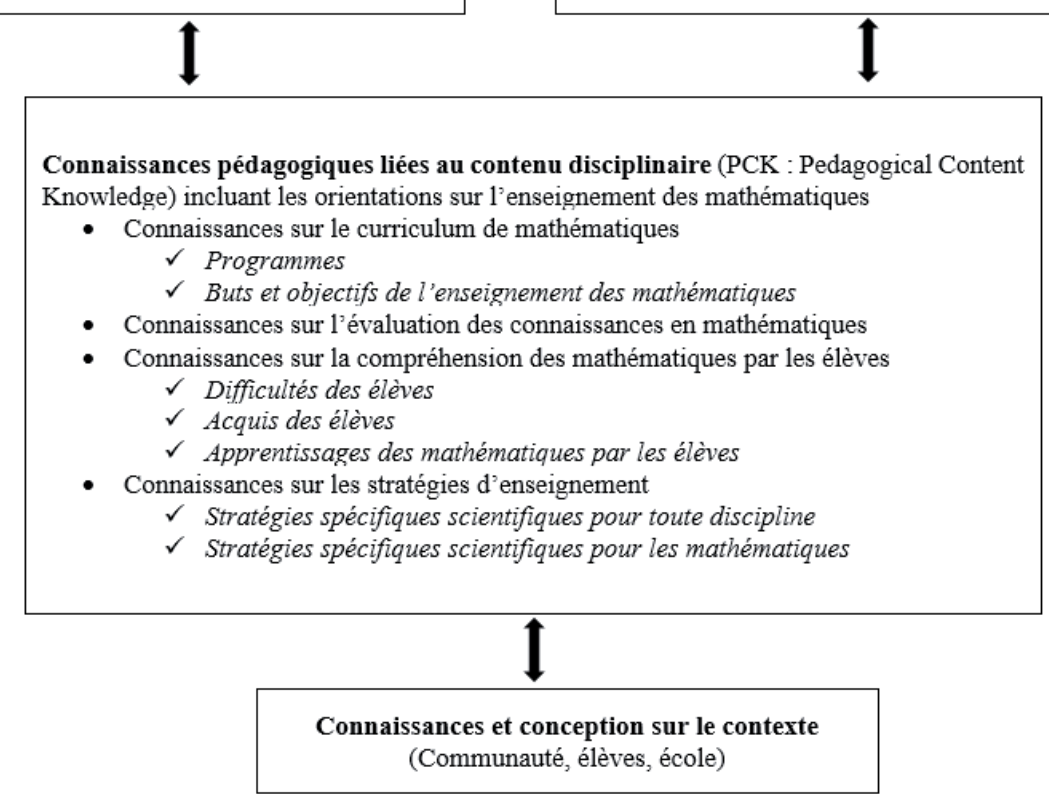

Figure 1

Domaines de connaissances des enseignants, adaptés par nous-mêmes à partir du modèle de Magnusson et al. (1999) 


\section{Méthodologie}

Les recherches sur les connaissances professionnelles des enseignants utilisent différentes approches méthodologiques en fonction des objectifs poursuivis et des contextes spécifiques. En effet, les connaissances des enseignants peuvent être étudiées à partir de leurs pratiques déclarées par des questionnaires ou des interviews (Abell, 2007) ou à partir d'un dispositif d'observation de leurs actions (Bécu-Robinault, 2007; Cross, 2011; Jameau, 2015). Dans notre cas, nous avons choisi d'étudier les connaissances en conduisant des entretiens avec les enseignants de mathématiques sur leurs pratiques enseignantes. Le choix de procéder par entretien est motivé par la possibilité de reformuler les questions non comprises, et l'obtention de réponses plus détaillées qu'à l'écrit. Nous avons ensuite été en mesure de produire un questionnaire diffusé plus largement (Ntwari, 2018).

\section{Caractéristiques des enseignants interviewés}

Nous avons conduit des entretiens auprès de 20 enseignants de mathématiques prestant en $7^{\mathrm{e}}$, $8^{\mathrm{e}}$ et $9^{\mathrm{e}}$ année de l'École Fondamentale. Ces enseignants ont été choisis au hasard et nous avons retenu ceux qui ont spontanément accepté d'être interviewés. Nous avons aussi retenu au hasard deux provinces scolaires, l'une en milieu rural, l'autre en milieu urbain (tableau 1).

Notre population comprend 10 enseignants travaillant dans 7 écoles en Bujumbura mairie (milieu urbain) et 10 enseignants travaillant dans 8 écoles en Bujumbura rural. Les milieux ruraux sont caractérisés par une population importante d'enseignants non qualifiés et une insuffisance d'équipements scolaires (UNESCO, 2012). Nous considérons qu'un enseignant est "ancien» dans l'enseignement s'il a déjà enseigné pendant plus de cinq ans; il est «nouveau» s'il a déjà enseigné pendant cinq ans maximum. Chaque enseignant est repéré par l'initiale de son prénom.

Notre échantillon est constitué de trois types d'institutions de formation initiale de l'enseignant :

1) FENM : Formation à l'Enseignement Non Mathématiques (Formation à l'enseignement autres que des mathématiques).

2) NFE : Non Formation à l'Enseignement. Exemples : Formation en section scientifique ou en sciences de la santé à l'école secondaire, formation en sciences économiques ou en Génie civil dans l'enseignement supérieur.

3) FEM : Formation à l'Enseignement des Mathématiques : Cas de l'École Normale Supérieure (section Mathématiques) et de l'Institut de Pédagogie Appliquée. 
Dans le tableau 1 qui suit, la colonne « $\mathbf{M}$ » désigne Milieu de travail de l'enseignant : $U$ = Urbain; $R$ = Rural, tandis que la colonne « $\mathbf{A}$ » parle de l'Ancienneté de l'enseignant: $\mathrm{A}=$ Ancien (si > 5ans); $\mathrm{N}$ $=$ Nouveau (si $\leq 5$ ans).

\section{Tableau 1}

Caractéristiques des enseignants interviewés

\begin{tabular}{|c|c|c|c|c|c|c|c|c|c|c|}
\hline \multirow[t]{3}{*}{ Milieu } & \multicolumn{5}{|c|}{ Institution de formation initiale } & \multicolumn{3}{|c|}{ Classe } & \multicolumn{2}{|c|}{ Ancienneté } \\
\hline & \multicolumn{2}{|c|}{ École secondaire } & \multirow{2}{*}{$\begin{array}{l}\text { École Normale } \\
\text { Supérieure } \\
\text { Section } \\
\text { Mathématiques }\end{array}$} & \multicolumn{2}{|c|}{ Université du Burundi } & \multirow[t]{2}{*}{$7 \grave{~}$} & & \multirow[t]{2}{*}{ 9è } & \multirow{2}{*}{$\leq 5$ ans } & \multirow[t]{2}{*}{$>5$ ans } \\
\hline & $\begin{array}{l}\text { École } \\
\text { Normale } \\
\text { secondaire }\end{array}$ & $\begin{array}{l}\text { Secondaire } \\
\text { (autres) }\end{array}$ & & $\begin{array}{l}\text { Institut de } \\
\text { Pédagogie } \\
\text { Appliquée/Maths }\end{array}$ & Autres & & & & & \\
\hline Urbain & 3 & 0 & 3 & 3 & 1 & 4 & 3 & 3 & 4 & 6 \\
\hline Rural & 0 & 4 & 4 & 0 & 2 & 2 & 4 & 4 & 4 & 6 \\
\hline Total & 3 & 4 & 7 & 3 & 3 & 6 & 7 & 7 & 8 & 12 \\
\hline $\begin{array}{l}\text { Statut de } \\
\text { l'institution }\end{array}$ & FENM & NFE & FEM & FEM & FENM & & & & & \\
\hline $\begin{array}{l}\text { Enseignant } \\
\text { citédans } \\
\text { l'article }\end{array}$ & «E» & $\begin{array}{l}\text { «B» } \\
\text { «D» }\end{array}$ & $\begin{array}{l}\text { «N } \\
\text { «P» } \\
\text { « } R »\end{array}$ & $\begin{array}{l}\text { "A» } \\
\text { «C» } \\
\text { «S» }\end{array}$ & «F» & & & & & \\
\hline
\end{tabular}

\section{Méthode et technique de recherche}

Dans notre étude, nous avons opté pour une «approche qualitative». Ce choix est lié à la volonté de mettre en évidence la manière dont les enseignants mobilisent leurs connaissances plutôt que de quantifier ces dernières et d'établir des corrélations (Paillé et Mucchielli, 2016). Notre recherche vise à comprendre la subjectivité des enseignants à travers leurs témoignages des expériences vécues (Poisson, 1983). L'entretien semi-directif a permis le recueil des données relatives à la manière dont les enseignants mobilisent différentes connaissances lors de la préparation et l'enseignement du cours de mathématiques, la part de formation initiale, de la pratique professionnelle et des formations continues comme sources de connaissances ainsi que les difficultés rencontrées, leurs origines et les solutions adoptées.

Nous avons réalisé une préenquête en menant un entretien exploratoire auprès d'une enseignante pour tester notre guide d'entretien et nous familiariser avec le terrain (Sauvayre, 2013). Les thèmes et les sous-thèmes étant compréhensibles et ne nécessitant donc pas de retouche, nous sommes passés à l'enquête auprès des 20 enseignants. Lors de nos entretiens, la semi-directivité a prévalu dans la mesure où nous invitions les sujets à s'exprimer sur les thèmes établis à l'avance; les sujets avaient toute la latitude de s'exprimer librement, mais nous les réorientions dans les thèmes à aborder à l'issue de chaque réponse en cas de besoin. Pendant 30 minutes en moyenne, chaque enseignant racontait comment il préparait et donnait ses cours de mathématiques et fournissait les raisons qui expliquaient ses choix. Ils explicitaient aussi les ressources mobilisées pour construire et enseigner leurs cours ainsi 
que les limites auxquelles ils se heurtaient. Les entretiens portaient sur l'ensemble des leçons enseignées durant l'année en cours.

Après la collecte des données, nous avons procédé à une analyse de contenu, traitement «manuel» comme méthode d'analyse qualitative d'entretiens qui permet la matérialisation des données (Wanlin, 2007). Lordre de traitement des données suit celui des domaines de connaissances des enseignants tels que proposés par Magnusson et al. (1999) en les liant avec leurs sources ${ }^{1}$ ainsi que les caractéristiques personnelles de l'enseignant le cas échéant².

\section{Résultats}

\section{Mobilisation des connaissances dans les pratiques enseignantes}

Nous exposons ci-après les résultats de notre étude, en les organisant suivant les domaines de connaissances de Magnusson et al. (1999) présentés dans la Figure 1.

\section{Connaissances et conceptions sur le contenu disciplinaire en mathématiques}

Nous tenons tout d'abord à préciser qu'il n'a pas été aisé de mettre en évidence la manière dont les enseignants mobilisent leurs connaissances disciplinaires en préparant leurs cours. En effet dix-neuf enseignants sur vingt interviewés ont indiqué qu'«ils ne préparent pas» le cours de mathématiques comme ils le faisaient auparavant dans leur cahier de préparation. Ainsi, l'enseignante $" \mathbf{C} »^{3}$ nous dit que ce ne sont pas les professeurs qui choisissent des contenus et des méthodes, mais que tout est planifié, consigné, que la matière est déjà préparée dans le guide de l'enseignant :

En fait, ce ne sont pas les profs qui choisissent des contenus, il y a des livres quion nous a donnés, là-dedans il y a des contenus et des démarches méthodologiques, nous essayons de suivre cela; tout est planifié dans les livres. ${ }^{4}$

De son côté, l'enseignant « $\mathbf{A} »$, qui a reçu la même formation que « $\mathbf{C}$ », affirme s'appuyer aussi sur son expérience pour préparer à sa façon les contenus de ses cours : "Je dirais que les nouveaux manuels quion nous a donnés, ne sont pas bien conçus, des fois je suis obligé de préparer le contenu-matière de ma façon, je vais au-delà de ce qui est préru, j'ajoute des notions». Pour sa part, le sujet « $\mathbf{F} »^{5}$ justifie pourquoi il ne suit pas textuellement ce qui est proposé dans le guide de l'enseignant :

Il y a des erreurs, généralement ces erreurs s'observent dans les guides de l'enseignant, parce quion $y$ met des prétendues réponses, et ces réponses sont souvent fausses, les opérations sont mal menées, elles ne sont pas bien faites. Mais aussi dans les livres des élèves il y a des erreurs: on pose des questions souvent illogiques, qui ne sont pas bien claires, on y trouve des réponses qui ne sont pas correctes dans le guide de l'enseignant.

Nous constatons que la perception des erreurs dans les manuels n'est pas spécifique aux enseignants initialement formés en mathématiques. En effet, même les enseignants non initialement formés en mathématiques constatent des erreurs sur le plan du contenu. Par conséquent, nous avons cherché à savoir quelle est la part de la formation initiale dans la mobilisation de connaissances disciplinaires. Notre étude indique que ce sont surtout les enseignants qui nont pas de formation initiale en 
mathématiques qui expriment avoir des connaissances mathématiques insuffisantes. Ils compensent en s'appuyant notamment sur leur expérience: "Non non, je n'ai pas eu de formation en maths mais c'est en forgeant quion devient forgeron» $(« \mathbf{D} »)^{6}$; «Oui, avec l'expérience, avec dix-sept ans, alors j'ai une expérience pour ne pas dire que je suis à la bauteur de tout» $(« \mathbf{E} »)^{7}$. Les enseignants qui affirment recourir à des savoirs mathématiques présentés en formation initiale sont ceux qui ont reçu une formation disciplinaire en mathématiques: "Ah oui, il y a des éléments par exemple dans le cours de trigonométrie [en formation initiale], tout ça, ça se trouve dans le programme» $(« \mathbf{P} »)^{8}$. C'est le cas aussi de « $\mathbf{N} »$ qui a la même formation que «P»: «A l'ENS [École Normale Supérieure], $j$ 'ai appris les maths, oui il y en a [savoirs mathématiques acquis en formation initiale] par exemple en trigonométrie, et des notions sur les équations».

À l'instar de Loewenberg Ball et al. (2008), nous faisons le constat que les savoirs présentés en formation initiale, notamment en trigonométrie et sur les équations, aident les enseignants à préparer et à enseigner leurs leçons. Notre analyse met aussi en évidence que ce sont surtout les enseignants formés dans des institutions de formation initiale d'enseignants qui utilisent des connaissances disciplinaires dans leur enseignement.

L'étude des connaissances des enseignants à partir du cadre théorique des PCK a permis d'éclairer aussi la manière dont les enseignants perçoivent la spécificité de l'enseignement des mathématiques comparativement à d'autres disciplines d'enseignement. En effet, treize enseignants sur vingt estiment que les mathématiques sont "plus exigeantes» que les disciplines littéraires. Les arguments avancés sont qu'en mathématiques, il y a une procédure, les leçons s'enchaînent de manière logique. Cela exige un "sérieux» dans la préparation des leçons selon les termes des enseignants : "L'enseignant de maths doit être actif, doit être plus compétent que les autres parce que les maths prennent beaucoup de temps pour la préparation, pour l'enseignement et pour faire les activités prévues, le temps de faire comprendre la matière aux élèves, tout prend beaucoup de temps» («F $\mathbf{F}) ;$; Les maths c'est très très difficile, tu dois préparer sérieusement, parce que si devant un enfant tu vas lui dire que $1+1=3$; ça ne marche pas, mais en français on peut discuter $($ ( $\mathbf{E} ») ;$ «Oui c'est un peu dur si on enseigne les maths, en maths on fait une procédure, on ne peut pas sauter une étape [il y a une chronologie logique des leçons]. Le problème particulier en maths est qu'il y a une suite logique si on saute une matière on ne peut pas continuer» ( $\mathbf{D} »)$.

Enfin, la perception que l'enseignant a de la discipline qu'il enseigne peut influencer sa façon de préparer ses leçons, notamment "en remplaçant des concepts non familiers aux élèves par des synonymes» (propos évoqués de manière similaire par huit enseignants). Ces enseignants pensent que les mathématiques seraient plus exigeantes que les disciplines littéraires, ce qui les obligerait à prendre du temps pour les préparer, et ce, indépendamment de la formation et de l'expérience de chacun. Néanmoins, en comparaison avec d'autres disciplines scientifiques, certains enseignants pensent que les mathématiques seraient plus faciles à enseigner. La raison est que les mathématiques sont une discipline à part, alors que les autres disciplines scientifiques sont regroupées dans ce qu'on appelle «domaine des sciences et technologie» dans les curricula: "En sciences, biologie, chimie, physique, on les a regroupées ensemble, raison pour laquelle un enseignant du domaine des sciences et technologie éprouve plus de difficultés quiun enseignant de maths, les maths sont une discipline à part» (« $\mathbf{R} »)$; «Les maths comparativement aux autres disciplines, n'exigent pas beaucoup comme les sciences et technologie où on a un mélange de beaucoup de choses, bio, physique, beaucoup de choses plus compliquées que les maths» (« $\underline{\mathbf{S}}$ ). 


\section{Connaissances pédagogiques et conceptions sur la gestion d'une classe}

Gérer une classe est une activité complexe et difficile : l'enseignant doit s'adapter à une réalité sans cesse changeante (Archambault et Chouinard, 2009). La gestion de la classe dépend de son contexte :

À cause des effectifs des élèves, des fois, je suis obligé de ne pas circuler comme je le voudrais, parce qu'il n'y a pas de passage, je ne parviens pas à atteindre chaque élève comme je le voudrais, je suis obligé de rester dans un coin. J'introduis la leçon, je la développe, je donne l'application et les élèves posent des questions $« \mathbf{A} »)^{9}$.

Dans les institutions de formation des enseignants, les étudiants bénéficient d'une formation pédagogique à travers des cours relatifs à la pédagogie et font un stage d'enseignement, alors que dans d'autres institutions, ce type de formation n'est pas inscrit dans les programmes. Mais la formation initiale n'est pas la seule à influencer les pratiques; le rôle des formations continues est important pour l'enseignant $« \mathbf{S} »^{10}$ :

D'après encore une fois les directives de l'École Fondamentale, on nous dit ceci : les activités vous les débattez ensemble, la classe est divisée en groupes, en forme de U c'est ça qui est proposé; si une question nécessite un débat, les élèves discutent en groupe.

En parlant de «directives», nous comprenons que lors des formations continues, les formateurs exposent aux enseignants les pratiques pédagogiques préconisées pour la mise en œuvre du curriculum en classe. Le poids non négligeable des prescriptions du curriculum dans la gestion pédagogique apparaît chez l'enseignant « $\mathbf{S}$ » quand il parle de «... directives...». Nous pensons que ce poids est surtout perceptible quand le curriculum est encore nouveau et que les enseignants essaient avant tout de respecter ce qui est prévu au lieu de se lancer dans des initiatives individuelles qui n'auraient pas encore fait leurs preuves.

\section{Connaissances pédagogiques liées au contenu disciplinaire «PCK»}

Des connaissances sur le curriculum

Les enseignants interviewés semblent avoir des connaissances sur le curriculum, mais ne l'exploitent pas de la même façon : «Normalement, c'est bien préparé dans les livres, on n'a pas de choix on doit suivre ce qui est donné dans les livres» ( $\left.\mathbf{D} »^{11}\right)$;

On ne peut pas dépasser ce qui se trouve dans les livres des élèves [dépasser veut dire aller au-delà] parce que les notes se trouvent dans le livre. Je me prépare à la matière pour la maîtriser, et je vais la dispenser. Parce que la trajectoire est déjà tracée, la manière de poser des questions, tout est préru ce n'est pas comme dans l'ancien système. $\left(« \mathbf{F} »^{12}\right)$

À travers les propos de ces enseignants ayant des qualifications et des expériences professionnelles variées, nous constatons qu'ils mettent en application les indications du curriculum tel qu'il est construit en matière de contenu disciplinaire et de procédures d'une leçon. Cette catégorie de PCK, introduite par Magnusson et al. (1999), semble donc adaptée pour mettre en évidence le rôle spécifique de ces connaissances. Par ailleurs, le fait que nous retrouvons souvent l'expression «Il ne faut pas aller audelà» peut s'expliquer dans le contexte de mise en œuvre d'un nouveau curriculum qui n'a pas encore été suffisamment appliqué; les formations continues semblent jouer un rôle important concernant les 
orientations curriculaires, car elles véhiculent également des messages d'obligation, créant parfois un sentiment de déprofessionnalisation (Wittorski et Roquet, 2013).

\section{Évaluation des connaissances et de la compréhension des mathématiques par les élèves}

L'évaluation des apprentissages permet à l'enseignant de juger de l'atteinte des objectifs d'une leçon. Même si tous les enseignants interviewés sont conscients du rôle que jouent les mathématiques, que ce soit dans la vie quotidienne ou dans les autres sciences, nous constatons à travers leurs propos que ces derniers montrent ou parlent de l'utilité de l'apprentissage des mathématiques plus que de l'évaluation de la compréhension des savoirs mathématiques. En effet, l'enseignant « $\mathbf{C}$ » fait partie des huit enseignants qui disent que les élèves comprennent l'utilité des mathématiques à travers des exercices d'application : "C'est ça quion précise dans le fondamental, on prévoit des exercices, des problèmes de la vie courante, quills doivent résoudre en utilisant les mathématiques».

Quatorze enseignants sur les vingt interviewés affirment qu'ils n'arrivent pas à évaluer si les élèves ont compris l'utilité de l'apprentissage des mathématiques. Les enseignants parlent de l'importance de cet apprentissage, mais n'arrivent pas à trouver des situations où les élèves peuvent le découvrir par eux-mêmes. En revanche, certains enseignants demandent aux élèves de donner eux-mêmes des exemples d'application d'une leçon de mathématiques donnée dans la vie courante : «Si jenseigne les nombres entiers, j'interroge les élèves quelle est l'utilité des nombres entiers, jessaie de demander aux élèves de donner des exemples de la vie courante pour évaluer s'ils ont compris» («D»). En outre, ce sont surtout les enseignants non initialement formés à l'enseignement qui semblent dispenser les mathématiques comme une discipline purement scolaire. Un enseignant explique que les élèves constateront plus tard l'importance d'avoir appris les mathématiques: "L'objectif on le voit à long terme, parce que nous aussi on apprenait les maths comme ça comme une discipline. Mais on voit l'utilité après l'école» ( $\mathbf{N} »)$. Un autre enseignant estime qu'il est difficile de démontrer l'utilité de l'apprentissage des mathématiques, car, selon lui, il existe des choses qu'il faut donner comme telles sans toutefois les démontrer: "Dans la matière il y a des choses quion n'expose pas. Par exemple dire à un élève qu'un nombre exposant 0 égale 1 c'est très compliqué, je leur dis de prendre comme ça» («B»). Cette affirmation peut être interprétée comme étant liée à l'insuffisance des connaissances mathématiques de cet enseignant. Ce résultat fait apparaître la nécessité, déjà pointée par Loewenberg Ball et al. (2008), de disposer de connaissances sur les mathématiques, au-delà des connaissances des mathématiques.

Enfin, quatorze enseignants sur les vingt interviewés affirment qu'ils n'arrivent pas à évaluer si les élèves ont compris l'utilité de l'apprentissage des mathématiques : "Je n'arrive pas à évaluer si les élèves comprennent l'utilité de l'apprentissage des maths» (« $\mathbf{C} »)$. Une explication possible à ce résultat tient au fait que dans le curriculum de mathématiques, il n'est pas mentionné que l'enseignant doit vérifier si les élèves comprennent ou pas l'utilité de l'apprentissage des mathématiques. Ce qui reste visible, c'est que les enseignants ne semblent pas trop penser à des pratiques d'évaluation qui ne sont pas inscrites dans le curriculum étant donné que même les questions d'évaluation des connaissances y sont incluses : «Au fait, dans ces jours avec l'arrivée de l'École Fondamentale, il n'y a pas beaucoup à penser, à chercher» (« $\mathbf{S} »)$. 


\section{Connaissances et conceptions sur le contexte}

La dernière catégorie de connaissances étudiées relève du contexte. Cette catégorie, intégrée au modèle des PCK par Magnusson et al. (1999), vise à mettre en évidence l'influence des conditions particulières de l'établissement et/ou de la classe sur le travail de l'enseignant. Dans notre cas, huit enseignants mentionnent les conditions d'exercice difficiles au sein de leurs classes. Ils dénoncent entre autres les effectifs élevés dans leurs classes, ce qui rend les évaluations complexes :

Comme c'est la première fois que j'enseigne deux classes parallèles, je vois qu'il me sera très difficile de donner beaucoup d'évaluations, donner sept interrogations qui sont exigées au cours d'un trimestre, ce sera difficile puisque j'ai d'autres évaluations à faire en dessin artistique. («B

Si certains enseignants estiment que le nombre maximal d'élèves par classe est de 50, d'autres, en raison peut-être des pratiques préconisées et du niveau des élèves, ne partagent pas cet avis :

Bon, moi, je pense que, je vais commencer par l'avis de l'UNESCO, mais je ne partage pas l'avis avec l'Unesco, l'Unesco dit qu'une classe est élevée à partir de cinquante élèves. Pendant les surveillances, à partir de 50 élèves on donne deux surveillants. Mais à mon avis, il faut au maximum trente élèves pour pouvoir suivre chaque élève. (« $\mathbf{R} »)$

Par ailleurs, si l'effectif d'une classe influence les pratiques pédagogiques, l'origine socio-économique des élèves est aussi prise en compte dans les pratiques enseignantes: "Les élèves qui n’ont pas de livres, on essaie de regrouper les élèves» (« $\mathrm{S} »)$. Les enseignants essaient de s'adapter au contexte de leur établissement en matière de disponibilités de manuels scolaires, surtout en milieu rural. Les élèves n'ayant pas tous des livres à leur disposition, les devoirs à domicile sont copiés au tableau, ce qui oblige l'enseignant à réduire le temps de la leçon : "Les élèves ne font pas des devoirs à domicile, les devoirs à domicile sont notés au tableau, ça nous prend évidemment du temps; ce qui entraîne des retards» («R»).

\section{Origine des connaissances des enseignants}

À l'instar d'une recherche précédemment conduite par Bécu-Robinault (2007), nous avons souhaité mettre en évidence l'origine des connaissances des enseignants. L'analyse des données recueillies nous permet de mettre en évidence que la principale ressource exploitée par les enseignants pour préparer et enseigner les mathématiques est le guide de l'enseignant et le manuel des élèves comme nous le constatons dans le tableau 2 qui suit.

\section{Tableau 2}

Origine des connaissances mobilisées par les enseignants

Source de connaissances

Nombre d'enseignants

\begin{tabular}{|l|c|}
\hline Guide de l'enseignant et manuel de l'élève & 19 \\
\hline Contexte de la classe & 5 \\
\hline Expérience professionnelle de l'enseignant & 2 \\
\hline Acquis de la formation initiale & 3 \\
\hline Consultation des autres collègues & 3 \\
\hline Comparaison des contenus entre l'ancien et le nouveau programme & 3 \\
\hline Internet & 1 \\
\hline Bibliothèque & 1 \\
\hline
\end{tabular}


Les résultats mettent en évidence que 19 enseignants sur 20 exploitent le contenu du guide de l'enseignant/manuel des élèves pour préparer et donner un cours. Néanmoins, certains enseignants choisissent de s'appuyer sur d'autres sources comme l'ancien programme de mathématiques. C'est ce qu'exprime notamment " $\mathrm{C}$ » : "Si je prends les livres du nouveau système fondamental, je constate qu'il y a des exercices qui n'ont pas d'importance, alors je recours aux livres de l'ancien système pour compléter donc la matière». Ainsi, ils peuvent mettre en œuvre des pratiques qui ne sont pas exactement celles préconisées lors des formations continues : "Je ne suis pas satisfaite des méthodes quion nous a apprises, non je ne suis pas satisfaite. Cette méthode [la méthode interactive] ne va pas pour les élèves» («N»).

En plus du contenu des formations qui nétait visiblement pas consistant, les enseignants ne sont pas satisfaits des compétences des formateurs. L'enseignant « $\mathrm{S} »$ dit :

Au niveau des formateurs, il y avait même quelqu'un qui avait un niveau inférieur à nous, c'est une dame qui a ENS 3 [Formé à l'ENS pendant trois ans], mais le problème n'est pas là, le problème est que pendant les séances de formation, elle-même ne savait pas grand-chose.

Le jugement d'incompétence d'un formateur peut aussi s'établir lorsque le formateur n'est pas du même domaine de formation que les enseignants formés, comme le souligne l'enseignant « $\mathrm{S}$ » : $U n$ biologiste ne peut pas former les mathématiciens, il y a des difficultés dans la mise en cuuvre des méthodes apprises, ce n'est pas la formation qui m'a outillé, on ne nous a pas dit grand-chose». Néanmoins, malgré la perception globalement négative des formations continues reçues jusqu'alors, les enseignants interviewés souhaitent en bénéficier davantage. C'est ce qu'exprime «N» : "Nous avons besoin d'autres formations sur l'École Fondamentale parce que nous avons une formation à l'enseignement à l'école primaire».

Au regard des résultats obtenus, il semble que les formations continues suivies par les enseignants ne constituent pas une source très enrichissante pour leurs pratiques. L'une des principales raisons est l'incohérence entre les besoins et les attentes des enseignants (Merle et Sensevy, 2001). Une analyse préalable des besoins des enseignants en situation de pratique professionnelle semble constituer une des voies d'amélioration de l'efficacité de ces formations.

\section{Discussion}

Notre étude s'est donné pour objet d'étudier la manière dont les enseignants mobilisent leurs connaissances professionnelles dans la préparation et la mise en œuvre du cours de mathématiques à l'École fondamentale burundaise ainsi que les origines possibles de ces connaissances. À ces fins, nous avons choisi un cadre théorique relatif aux connaissances professionnelles. Parmi les nombreuses adaptations du cadre originellement présenté par Shulman (1986), nous avons choisi celui de Magnusson et al. (1999). Ce cadre est adapté au contexte de mise en œuvre d'un nouveau curriculum, il prend en considération la gestion et l'animation de la classe dans ce contexte et surtout il s'intéresse à l'évaluation des apprentissages. Nous avons réalisé et transcrit 20 entretiens auprès d'enseignants exerçant dans différents contextes et caractérisés par une variété de profils de formation. Concernant la manière dont les enseignants mobilisent leurs connaissances, notre étude dévoile que les enseignants se réfèrent aux orientations curriculaires, mais que le suivi de ces dernières dépend de l'appréciation de l'enseignant quant aux caractéristiques particulières de sa propre classe dont il reste spécialiste. Nous avons également observé que, malgré la reconnaissance de l'utilité des mathématiques dans la 
vie quotidienne, et en dépit de la formation initiale et de l'ancienneté de chacun, une grosse majorité des enseignants déclare ne pas pouvoir démontrer cette utilité à leurs apprenants. Plus encore, ils indiquent ne pas savoir comment évaluer si les élèves ont compris ou non l'utilité de l'apprentissage des mathématiques, ce qui pourrait avoir des conséquences sur la motivation des élèves. Une explication à ce sujet est que cette pratique n'est ni inscrite dans le curriculum ni dispensée en formation initiale ou continue. Enfin, les enseignants, même quand ils ne sont pas issus de formations académiques en mathématiques, reconnaissent des spécificités à cette discipline dont ils tiennent compte dans la préparation de leurs cours. Toutefois, une formation disciplinaire leur paraît nécessaire pour prendre du recul par rapport aux injonctions officielles, même si l'expérience professionnelle les aide à dépasser certaines lacunes.

En parallèle, nous avons également dégagé des origines possibles à ces connaissances. L'analyse des entretiens semi-directifs indique que les savoirs présentés en formation initiale jouent un rôle important dans la mobilisation des connaissances disciplinaires et pédagogiques en enseignant les mathématiques. Les enseignants non formés en mathématiques et non formés à l'enseignement, mais qui ont une ancienneté de plus de 5 ans dans l'enseignement, exploitent leur expérience professionnelle dans leurs pratiques. Pour les enseignants formés à l'enseignement, les connaissances pédagogiques émanent en partie des connaissances acquises en formation initiale, mais pas forcément sur l'enseignement des mathématiques. Les cours suivis en pédagogie et en méthodologie de l'enseignement les aident dans la gestion des classes. La quasi-totalité des 20 enseignants interviewés indique suivre les prescriptions du curriculum, peut-être parce qu'ils ne l'ont pas encore adapté en fonction des expériences quotidiennes accumulées. Même si les formations continues requièrent le suivi des prescriptions du curriculum, les enseignants formés en mathématiques et ayant une certaine expérience se donnent une marge de manœuvre pour ajouter des notions jugées essentielles, mais manquantes. Les formations continues organisées demeurent insatisfaisantes au regard de la durée (peu de temps), du contenu (des généralités et non des mathématiques) et des compétences des formateurs (des formateurs étrangers au domaine des mathématiques). Ces formations ne semblent donc pas répondre aux attentes des enseignants bénéficiaires. Si les formations continues insistent uniquement sur la mise en application du curriculum en vigueur, il est possible qu'en conséquence, tous les enseignants enseignent in fine les mêmes contenus de la même manière alors que les élèves ne sont pas les mêmes et que les contextes sont aussi divers que les classes. Ce dernier point pose une question quant au sentiment de déprofessionnalisation des enseignants.

\section{Conclusion}

Les résultats de notre étude sont issus de ce que les enseignants déclarent au sujet de leurs pratiques au travers d'entretiens et ultérieurement de questionnaires. Une étude complémentaire serait à envisager afin de mettre en évidence les connaissances effectivement mises en œuvre par les enseignants au cours de pratiques ordinaires. Une telle étude pourrait s'appuyer sur des observations de pratiques effectives en classe, qui seraient analysées au regard des contenus et des méthodes véhiculés par le curriculum de mathématiques. 
Parallèlement, nous avons mis en évidence le profil de formation initiale très varié des enseignants de mathématiques. Afin de répondre au mieux aux attentes des enseignants, il conviendrait de bâtir des formations continues sur la base de l'analyse des besoins réels des enseignants en poste. Ces premiers résultats nous ont d'ores et déjà conduits à diffuser une enquête sur l'évaluation de l'efficacité des formations professionnelles destinées aux enseignants, dont les résultats donnent à voir des leviers à actionner pour professionnaliser davantage ces formations. Cette enquête révèle qu'il est nécessaire d'améliorer la formation initiale des enseignants de mathématiques pour l'adapter aux pratiques et aux exigences actuelles de l'enseignement. Les résultats de notre étude indiquent que, s’il est nécessaire de renforcer les formations continues du point de vue des généralités sur l'enseignement, il conviendrait surtout de proposer des modules de formation orientés sur les contenus mathématiques et sur la méthodologie de l'enseignement des mathématiques. Afin d'accompagner au mieux les enseignants dans leurs pratiques quotidiennes, il semble donc impératif de proposer des formations fondées sur les résultats de la recherche en didactique des mathématiques et ses applications, en lien avec les prescriptions officielles au Burundi.

\section{Notes}

1 Le curriculum, formation initiale et continue, expérience professionnelle dans l'enseignement, ancien programme de mathématiques, etc.

2 Institution de formation initiale, diplôme, section suivie et ancienneté dans l'enseignement.

3 Enseignante en $9^{\text {ème }}$ depuis trente ans, formée à l'enseignement des mathématiques à l'Université du Burundi (FEM).

4 Les livres désignent le guide de l'enseignant et manuels pour élèves.

5 Elle enseigne en $8^{\text {ème }}$ depuis deux ans, formée en sciences de l'éducation à l'Université du Burundi (FENM).

6 Formé en sciences de la santé, ancien de 18 ans dans l'enseignement en $9^{\text {ème }}$ (NFE).

7 Enseignant en $7^{\text {tème }}$ année depuis 14 ans, diplômé des humanités générales en section scientifique (NFE).

8 Il enseigne en $7^{\text {ème }}$ depuis trois ans, formé à l'École Normale Supérieure, section mathématiques (FEM).

9 Formé à l'enseignement, ancien de 32 ans d'enseignement.

10 Formé à l'Institut de Pédagogie Appliquée/Mathématiques (FEM), ancien de 10 ans dans l'enseignement.

11 Formé en sciences de la santé, ancien de 18 ans dans l'enseignement en $9^{\text {ème }}$ (NFE).

12 Il enseigne en $8^{\text {ème }}$ depuis deux ans, formé en sciences de l'éducation à l'Université du Burundi (NFEM).

\section{Références}

Abell, S. K. (2007). Research on science teacher knowledge. Dans S. K. Abell et N. G. Lederman (dir.), Handbook of research on science education ( $1^{\mathrm{e}}$ éd., p. 1105-1149). Mahwah, NJ: Lawrence Erlbaum Associates.

Archambault, J. et Chouinard, R. (2009). Vers une gestion éducative de la classe (3éd.). Montréal : Gaëtan Morin éditeur.

Bécu-Robinault, K. (2007). Connaissances mobilisées pour préparer un cours de sciences physiques. Aster, 45, 165-188. https://doi.org/10.4267/2042/16822

Brau-Antony, S. et Hubert, B. (2014). Curriculum en Éducation Physique et Sportive et évaluation certificative au baccalauréat. Questions Vives. Recherches en éducation, 22. https://doi.org/10.4000/questionsvives.1596

Carlsen, W. (1999). Domains of teacher knowledge. Dans J. Gess-Newsome et N. Lederman (dirs.), Examining pedagogical content knowledge (1 éd., vol. 6, p. 133-144). Dordrecht : Springer. https://doi.org/10.1007/0-306-47217-1 5

Cochran, K. F., DeRuiter, J. A., et King, R. A. (1993). Pedagogical content knowing: An integrative model for teacher preparation. Journal of teacher Education, 44(4), 263-272. https://doi.org/10.1177/0022487193044004004

Cross, D. (2011). Action conjointe et connaissances professionnelles de l'enseignant. Éducation et didactique, 4(3), 39-60. https://doi.org/10.4000/educationdidactique.850 
Demeuse, M. (2013). Elaborer un curriculum de formation et en assurer la qualité. Dans F. Parent et J. Jouquan (dir.), Penser la formation des professionnels de la santé. Une perspective intégrative (1 éd., p. 315-330). De Boeck.

Djibo, F., et De Rivière-du-Loup, C. (2017). L'efficacité de la formation continue des enseignants du primaire : le cas du Burkina Faso. Formation et profession, 25(2), 35-48. http://dx.doi.org/10.18162/fp.2017.330

Grossman, P. L. (1990). The making of a teacher: Teacher knowledge and teacher education (1e éd.). New York: Teachers College Press.

Jameau, A. (2015). Les connaissances professionnelles des enseignants et leur évolution à travers une analyse de l'activité. Une étude de cas en physique au collège. Éducation et didactique, 9 (1), 9-31. https://doi.org/10.4000/educationdidactique. 2140

Jonnaert, P. (2011). Curriculum, entre modèle rationnel et irrationalité des sociétés. Revue internationale d'éducation de Sèvres, 56, 135-145. https://doi.org/10.4000/ries.1073

Jorro, A. (2014). Dictionnaire des concepts de la professionnalisation (1 ${ }^{\mathrm{e}}$ éd.). Louvain-la-Neuve, Belgique : De Boeck supérieur. https://doi.org/10.3917/dbu.devel.2013.02

Leroux, M. et Vivegnis, I. (2019). Cercle pédagogique et analyse de cas : dispositifs complémentaires de réflexion collective au cœur de la formation initiale des enseignants. Formation et profession, 27(2), 58-73.

$\underline{\text { http://dx.doi.org/10.18162/fp.2019.506 }}$

Loewenberg Ball, D., Thames, M. H. et Phelps, G. (2008). Content knowledge for teaching: What makes it special ? Journal of teacher education, 59(5), 389-407. https://doi.org/10.1177/0022487108324554

Magnusson, S., Krajcik, J. et Borko, H. (1999). Nature, sources, and development of pedagogical content knowledge for science teaching. Dans J. Gess-Newsome et N.G. Lederman (dir.), Examining pedagogical content knowledge (1éd., vol. 6, p. 95-132). Dordrecht: Springer. https://doi.org/10.1007/0-306-47217-1 4

Merle, P. et Sensevy, G. (2001). Une modalité de régulation des pratiques professionnelles : la recherche d'une articulation entre offre et demande dans la formation continue des enseignants. Éducation et sociétés, 8(2), 27-48. https://doi.org/10.3917/es.008.0027

Ministère de l'éducation, de l'enseignement supérieur et de la recherche scientifique du Burundi. (2014, septembre). Curriculum de mathématiques à École Fondamentale. Guide de l'enseignant, Cycle 4, 2014.

Ministère de l'éducation, de l'enseignement supérieur et de la recherche scientifique du Burundi, Bureau de la Planification de l'Éducation (2017, octobre). Répartition des enseignants par qualification, 2017.

Morine-Dershimer G. et Kent T. (1999) The complex nature and sources of teachers' Pedagogical knowledge. Dans J. GessNewsome et N.G. Lederman (dir.), Examining Pedagogical Content Knowledge (1 ${ }^{\mathrm{e}}$ éd., vol. 6, p. 21-50). Dordrecht : Springer. https://doi.org/10.1007/0-306-47217-1 2

Ntwari, I. (2018). Connaissances professionnelles mobilisées et besoins des enseignants pour la mise en œuvre du curriculum de mathématiques au 4ème cycle de l'École Fondamentale au Burundi (Élèves de 12 à 15 ans). [Thèse de doctorat, Université de Lyon]. HAL. https://tel.archives-ouvertes.fr/tel-02076318

Paillé, P. et Mucchielli, A. (2016). L'analyse qualitative en sciences humaines et sociales (4éd.). Paris : Armand Colin. https://doi.org/10.3917/arco.paill.2016.01

Paquay, L. (2007). À quoi bon un curriculum de qualité s'il ne transporte par les pratiques enseignantes! Dans M. Behrens, La qualité en éducation (1 $1^{\mathrm{e}}$ éd., p. 57-98). Presses de l'Université du Québec.

Paquay, L., Marguerite, A., Charlier, E. et Perrenoud, P. (2012). Former des enseignants professionnels : quelles stratégies? Quelles compétences? ( $4^{\mathrm{e}}$ éd.). Bruxelles : De Boeck.

Perrenoud, P. (1993). Curriculum : le formel, le réel, le caché. Dans J. Houssaye (dir.), La pédagogie : une encyclopédie pour aujourd'hui (1 éd., p. 61-76). Paris : ESF.

Poisson, Y. (1983). L'approche qualitative et l'approche quantitative dans les recherches en éducation. Revue des sciences de l'éducation, 9(3), 369-378. https://doi.org/10.7202/900420ar

Sauvayre, R. (2013). Les méthodes de l'entretien en sciences sociales. Paris : Dunod. https://doi.org/10.3917/dunod.sauva.2013.01

Schwille, J., Dembélé, M. et Schubert, J. (2007). Former les enseignants : politiques et pratiques. Éditions de l'Unesco. http://www.iiep.unesco.org/fr/publication/former-les-enseignants-politiques-et-pratiques 
Shing, C. L., Saat, R. M. et Loke, S. H. (2015). The knowledge of teaching-Pedagogical content knowledge (PCK). Malaysian Online Journal of Educational Sciences, 3(3), 40-55.

Shulman, L. S. (1986). Those who understand: Knowledge growth in teaching. Educational researcher, 15(2), 4-14. https://doi.org/10.3102/0013189X015002004

Shulman, L. (1987). Knowledge and teaching: Foundations of the new reform. Harvard educational review, 57(1), 1-23. https://doi.org/10.17763/haer.57.1.j463w79r56455411

Smith, D. C. et Neale, D. C. (1989). The construction of subject matter knowledge in primary science teaching. Teaching and teacher Education, 5(1), 1-20. https://doi.org/10.1016/0742-051X(89)90015-2

Talbot, L. (2012). Les recherches sur les pratiques enseignantes efficaces, synthèse, limites et perspectives. Questions Vives. Recherches en éducation, 6(18), 129-140. https://doi.org/10.4000/questionsvives.1234

Unesco. (2012, mai). Analyse de la question enseignante au Burundi : Diagnostic et perspectives pour le renforcement des capacités du système éducatif. Unesco.

Wanlin, P. (2007). L'analyse de contenu comme méthode d'analyse qualitative d'entretiens : une comparaison entre les traitements manuels et l'utilisation de logiciels. Recherches qualitatives, 3(3), 243-272.

Wittorski, R. et Briquet-Duhazé, S. (2010). Développement professionnel d'enseignants du primaire et du secondaire. Travail en communautés, collaboration et partenariats pour le développement professionnel des enseignants. Éducation et formation, e-293, 211-222.

Wittorski, R. et Roquet, P. (2013). Professionnalisation et déprofessionnalisation : des liens consubstantiels. Recherche et formation, 72(1), 71-88.

https://doi.org/10.4000/rechercheformation.2038

\section{Pour citer cet article}

Ntwari, I., Bécu-Robinault, K. (2021). Mobilisation des connaissances professionnelles des enseignants dans la préparation et la mise en œuvre des cours de mathématiques à l'école fondamentale au Burundi. Formation et profession, 29(2), 1-16. http://dx.doi.org/10.18162/fp.2021.565 